\title{
29. LIGHT HYDROCARBONS IN SEDIMENTS OF DSDP LEG 44 HOLES ${ }^{1}$
}

John M. Hunt and Jean K. Whelan, Department of Chemistry, Woods Hole Oceanographic Institution, Woods Holes, Massachusetts

Seven gas samples obtained from cores taken on DSDP Leg 44 have been analyzed for hydrocarbons in the ethane through pentane range. Table 1 shows the distribution of these hydrocarbon gases. Values are expressed in ppm by volume of the individual hydrocarbon in the total gas. The yields of the heavier hydrocarbon gases were extremely low compared to other areas such as the Black Sea. For example, a Black Sea sample taken at 250 meters corresponding to the same depth as the first sample in Table 2 contained 50 $\mathrm{ppm}$ ethane, $5 \mathrm{ppm}$ propane, $2 \mathrm{ppm}$ butane, and 0.7 ppm pentane. Also, a Black Sea sample at 900 meters contained $1000 \mathrm{ppm}$ ethane, $200 \mathrm{ppm}$ propane, $70 \mathrm{ppm}$ butane, and $10 \mathrm{ppm}$ pentane.

\section{TABLE 1}

Concentration of $\mathrm{C}_{2}-\mathrm{C}_{5}$ Gases in Leg 44 Gas Samples

\begin{tabular}{lccccccc}
\hline \multicolumn{1}{c}{ Section } & Depth (m) & $\mathrm{C}_{2}$ & $\mathrm{C}_{3}$ & $i-\mathrm{C}_{4}$ & $n-\mathrm{C}_{4}$ & $i-\mathrm{C}_{5}$ & $n-\mathrm{C}_{5}$ \\
\hline 388A-5-6 (top) & 250 & 0.84 & 0.51 & 0.08 & 0.04 & 0.07 & 0.2 \\
$388 \mathrm{~A}-6-2$ (top) & 285 & 2.9 & 1.2 & 0.17 & 0.08 & 0.37 & 0.18 \\
$388 \mathrm{~A}-7-2-1$ & 292 & 0.51 & 0.31 & 0.07 & 0 & 0.06 & 0.08 \\
$388 \mathrm{~A}-7-3$ (top) & 294 & 0.12 & 0.18 & 0.04 & 0.01 & 0.02 & 0.09 \\
$388 \mathrm{~A}-7-4$ & 295 & 3.2 & 0.79 & 0.19 & 0.04 & 0.21 & 0.01 \\
$388 \mathrm{~A}-9-4$ & 314 & 2.7 & 0.85 & 0.20 & 0.05 & 0.20 & 0.12 \\
$391 \mathrm{C}-21-5$ & 1090 & 1.0 & 1.0 & 0.10 & 0.24 & 0.09 & 0.27 \\
\hline
\end{tabular}

The yield of $\mathrm{C}_{4}-\mathrm{C}_{7}$ hydrocarbons in sediments from Leg 44 holes was also low. For example, the yields of $\mathrm{C}_{4}-\mathrm{C}_{7}$ hydrocarbons at Site 391 in the Blake-Bahama Basin was about one-hundredth of the yields in the Angola Basin and Cape Basin off Africa and in the Black Sea at depths below 1000 meters. Data for these areas are plotted in Figure 1. The $\mathrm{C}_{4}-\mathrm{C}_{7}$ yields in all three of these latter areas is about $30,000 \mathrm{ng} / \mathrm{g}$ organic carbon at about 1000 meters depth compared to about $300 \mathrm{ng} / \mathrm{g} \mathrm{C}$ for Site 391. Yields at Hole 388A were also low. The results indicate that the type of organic matter in the samples analyzed is not conducive to forming oil, although the sediments may be capable of generating gas if buried deeper.

This work was supported by the National Science Foundation Grant OCE73-06575 A02.

${ }^{1}$ Woods Hole Oceanographic Institution Contribution No, 4186.

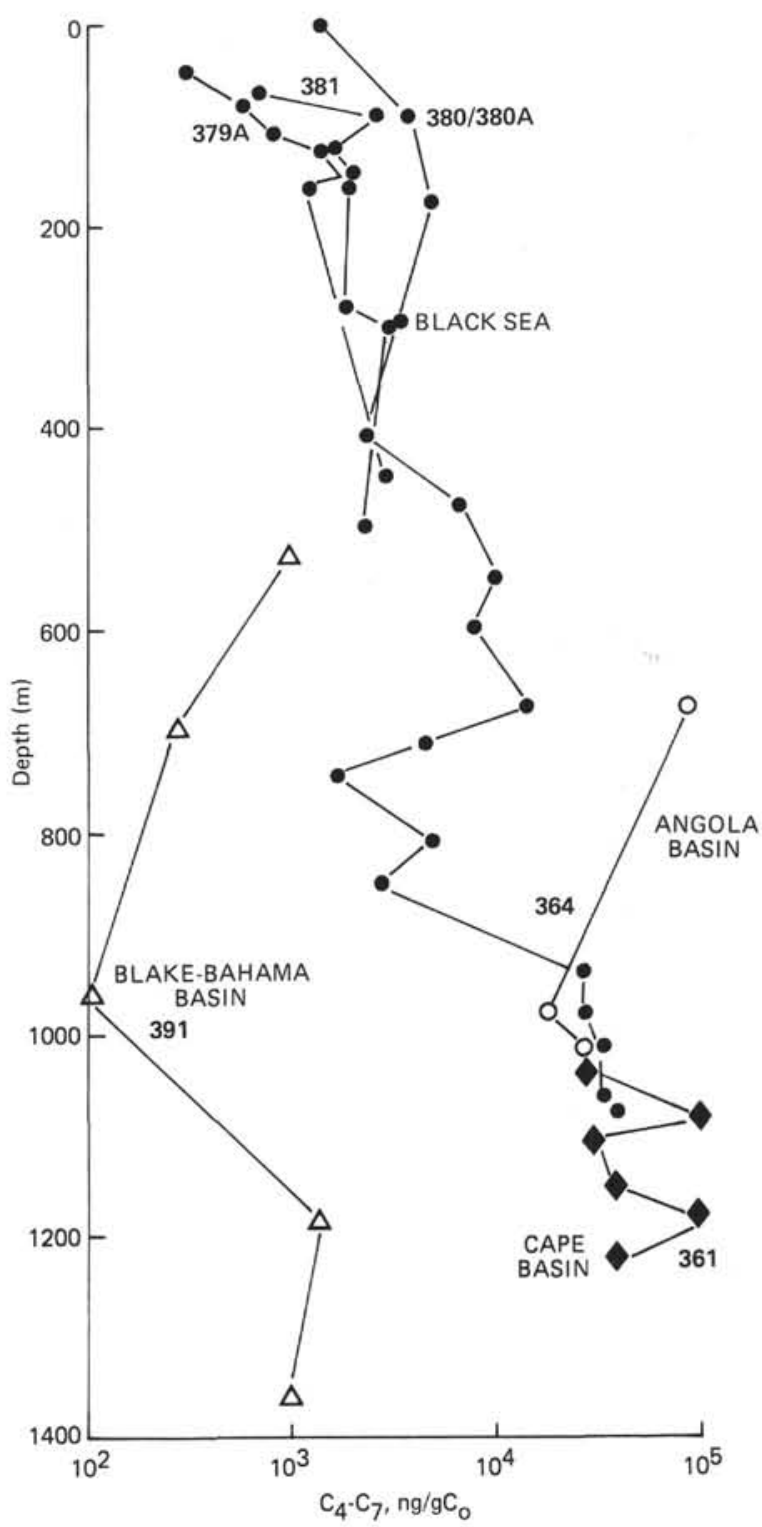

Figure 1. Yield of hydrocarbons in the butanes through heptanes range in nanograms per gram of organic carbon versus depth. 
TABLE 2

$\mathrm{C}_{4}-\mathrm{C}_{7}$ Hydrocarbons in Sediments of Leg 44 Holes

\begin{tabular}{|c|c|c|c|c|c|c|c|}
\hline \multirow{3}{*}{$\begin{array}{l}\text { Section } \\
\text { Depth (m) } \\
\text { Hydrocarbons }\end{array}$} & $388 \mathrm{~A}-5-0$ & $388 \mathrm{~A}-11-0$ & $391 \mathrm{~A}-13-0$ & $391 C-6-3$ & $391 C-12-4$ & $391 C-30-2$ & $391 C-48-2$ \\
\hline & 246 & 332 & 526 & 692 & 959 & 1185 & 1355 \\
\hline & \multicolumn{7}{|c|}{ Concentration (ng/g) } \\
\hline Isobutane & 0.01 & 0.31 & 0.24 & 0 & 0 & 0.98 & 0.26 \\
\hline$n$-Butane & 0.07 & 1.27 & 1.89 & 0.09 & 0.08 & 1.09 & 0.18 \\
\hline Isopentane & 0.45 & 1.69 & 0.29 & 0.05 & 0.09 & 1.61 & 0.08 \\
\hline$n$-Pentane & 0.08 & 0.25 & 0.36 & 0.03 & 0.03 & 1.30 & 0.04 \\
\hline 2, 2-Dimethylbutane & 0.05 & 0.26 & 0.83 & 0 & 0.03 & 0.04 & 0.02 \\
\hline Cyclopentane & 0.02 & 0.09 & 0.02 & 0.01 & 0.01 & 0.21 & $\operatorname{tr}$ \\
\hline 2, 3-Dimethylbutane & 0.03 & 0.23 & 0.74 & 0 & 0.01 & 0.21 & 0.04 \\
\hline 2-Methylpentane & 0.14 & 0.38 & 0.57 & 0.04 & 0.03 & 1.24 & 0.06 \\
\hline 3-Methylpentane & 0.05 & 0.15 & 0.13 & 0.01 & 0.01 & 0.22 & 0.08 \\
\hline$n$-Hexane & 0.08 & 0.30 & 0.17 & 0.01 & 0.01 & 0.83 & 0.05 \\
\hline Methylcyclopentane & 0.17 & 0.29 & 0.03 & 0.01 & 0.07 & 0.80 & 0.05 \\
\hline 2, 2-Dimethylpentane & 0.01 & 0.14 & 0.80 & 0 & 0 & 0.01 & 0.10 \\
\hline 2, 4-Dimethylpentane & 0.02 & 0.12 & 0.78 & 0 & 0 & 0.11 & 0.10 \\
\hline Cyclohexane & 0.12 & 0.40 & 1.22 & 0.02 & 0 & 0.33 & 0.01 \\
\hline 3, 3-Dimethylpentane & 0.10 & 0 & 0 & 0 & 0 & 0.01 & 0 \\
\hline 1, 1-Dimethylcyclopentane & 0.15 & 0.01 & $\operatorname{tr}^{\mathrm{a}}$ & 0 & 0 & 0.02 & 0 \\
\hline 2-Methylhexane & 0.49 & 0.13 & 0.09 & 0.32 & 0 & 0.21 & \\
\hline 2, 3-Dimethylpentane & 0.88 & 0.31 & 1.04 & 0.29 & 0.02 & 0.65 & 0.37 \\
\hline 3-Methylhexane & 0.14 & 0.34 & 0.25 & 0.08 & 0 & 0.37 & 0.09 \\
\hline 1-t-3-Dimethylcyclopentane & 0.06 & 0.2 & 0 & 0 & 0 & 0.16 & 0.03 \\
\hline 1-t-2-Dimethylcyclopentane & 0.04 & 0.12 & 0.01 & 0 & 0 & 0.13 & 0.06 \\
\hline$n$-Heptane & 0.14 & 0.54 & 0.26 & 0.14 & 0 & 0.87 & 0.07 \\
\hline Methylcyclohexane & 0.41 & 1.16 & 1.39 & 0.24 & $\operatorname{tr}$ & 0.30 & 0.07 \\
\hline Benzene & 0.74 & 0.04 & 0.11 & 0.01 & $\operatorname{tr}$ & 0.57 & 1.05 \\
\hline Toluene & 0.10 & 0.96 & 3.98 & 1.04 & $\operatorname{tr}$ & 0.52 & 0.08 \\
\hline Total (ng/g) & 4.6 & 9.7 & 15.2 & 2.4 & 0.39 & 12.8 & 2.9 \\
\hline
\end{tabular}

$a_{\mathrm{tr}}=$ trace. 